\title{
Asymptotic Distribution for the Sum and Maximum of Gaussian Processes
}

\author{
W. P. McCormick and Y. Qi \\ Department of Statistics \\ University of Georgia
}

\begin{abstract}
Previous work on the joint asymptotic distribution of the sum and maxima of Gaussian processes is extended here. In particular, it is shown that for a stationary sequence of standard normal random variables with correlation function $r$, the condition $r(n) \log n=$ $o(1)$ as $n \rightarrow \infty$ suffices to establish the asymptotic independence of the sum and maximum. Keywords: Gaussian process, Maximum, Sum, Weakly Dependent, Strongly Dependent.

AMS 1991 Subject Classification: Primary 60E70 Secondary 60F05, 60G15.

\section{Introduction}

The question of the joint asymptotic behavior of sums and maxima has interested researchers both for its theoretical challenges and its value in applications. Such a study relates to questions regarding the affect of extreme values on sums as well as quantifies the behavior of a system through its average and extreme values. For i.i.d. observations, the influential 1978 paper of Chow and Teugels delineates the asymptotic behavior of $\left(S_{n}, M_{n}\right)$ where $S_{n}=$ $\sum_{i=1}^{n} X_{i}$ and $M_{n}=\max _{1 \leq i \leq n} X_{i}$. They found, for example, that asymptotic independence of $S_{n}$ and $M_{n}$ occurs in only one instance, namely, that of the underlying distribution having membership in the domain of attraction (for sums) of the normal distribution and the domain of attraction (for maxima) of an extreme value distribution.

Recent work on this problem has focused on dependent sequences. We offer Hsing (1995) and Ho and Hsing (1996) as two recent papers on the problem and further references may be found by consulting these articles. The purpose of the present paper is to describe in greater generality than hitherto the asymptotic behavior of $\left(S_{n}, M_{n}\right)$ for Gaussian sequences and processes.
\end{abstract}


Consider, for example, two recent papers on the topic, Ho and Hsing (1996) and Ho and McCormick (1999). Both papers give satisfactory results for the case of strongly dependent Gaussian sequence. However, a gap exists for the case of stationary Gaussian sequences $\left\{X_{k}\right\}$ with correlation function $r(k)$ satisfying

$$
r(k) \log k=o(1) \text { as } k \rightarrow \infty .
$$

For a stationary Gaussian sequence with correlation function $r$, it is well known that condition (1.1) is sufficient to guarantee an extremal index equal to 1. In the Ho and Hsing (1996) paper, for the case of a stationary Gaussian sequence with $E X_{k}=0, E X_{k}^{2}=1$ and $r(k)=E X_{1} X_{k+1}$ satisfying (1.1), to conclude the asymptotic independence of $\left(S_{n}, M_{n}\right)$, it is additionally required that for $n \geq 1$ there exists a subset $I_{n} \subset\{1, \ldots, n\}$ with

$$
\frac{\log \left(1-\frac{\#\left(I_{n}\right)}{n}\right)}{\sqrt{\log n}} \rightarrow-\infty
$$

and

$$
\limsup _{n \rightarrow \infty} \sup _{s \in I_{n}} \frac{n \sum_{j=1}^{n} r(|s-j|)}{\sum_{i=1}^{n} \sum_{j=1}^{n} r(|i-j|)}<\infty .
$$

The condition expressed in (1.2) and (1.3) is rather weak and Ho and Hsing (1996) asks the question, does there exist a stationary Gaussian sequence satisfying (1.1) but for which (1.2) and (1.3) fail to hold. The following example shows that there does indeed exist such sequences. To that end let $r^{(k)}(n), n \geq 0, k \geq 1$ be a sequence of correlation functions such that for each fixed $k \geq 1$

$$
r^{(k)}(n)=I_{\{0\}}(n)-\frac{1}{2} I_{\{k\}}(n), \quad n \geq 0 .
$$

Note that $r^{(k)}$ is the correlation function for the moving average sequence

$$
X_{n}^{(k)}=\frac{1}{\sqrt{2}}\left[U_{n}-U_{n+k}\right], \quad n \geq 1,
$$

where $\left\{U_{n, n \geq 1}\right\}$ is an i.i.d. sequence of standard normal random variables. Let $p_{k}=$ $\frac{1}{k}-\frac{1}{k+1}, k=1,2, \ldots$ and define a correlation function by

$$
r(n)=\sum_{k=1}^{\infty} p_{k} r^{(k)}(n), \quad n \geq 0 .
$$

We shall show that a stationary Gaussian sequence with correlation function given in (1.4) provides the desired example.

First observe that

$$
\sum_{i=1}^{n} \sum_{j=1}^{n} r(|i-j|)=\sum_{k=1}^{n} \frac{1}{k} .
$$


Now observe that, for fixed $1 \leq s \leq n$,

$$
\begin{aligned}
& n \sum_{j=1}^{n} r(|s-j|) \\
= & n\left[1+\sum_{k=1}^{n-1} p_{k} \sum_{j=1}^{n}\left(-\frac{1}{2} I_{\{-k\}}(s-j)-\frac{1}{2} I_{\{k\}}(s-j)\right)\right] \\
= & n\left[1-\frac{1}{2} \sum_{k=1}^{n-s} p_{k}-\frac{1}{2} \sum_{k=1}^{s-1} p_{k}\right] \\
= & \frac{n}{2}\left[\frac{1}{n-s+1}+\frac{1}{s}\right] .
\end{aligned}
$$

Next observe that for all $n$ sufficiently large, if $I_{n} \subset\{1, \ldots, n\}$ is a sequence of subsets satisfying (1.2), then

$$
I_{n} \cap\left\{1, \ldots,\left[\epsilon_{n} n\right]\right\} \neq \phi
$$

where $\epsilon_{n}=(\log n)^{-2}$.

Therefore by (1.7) for all sufficiently large $n$, we have that there exists $s_{n} \in\left\{1, \ldots,\left[\epsilon_{n} n\right]\right\} \cap$ $I_{n}$.

For such $s_{n}$, we have by (1.5) and (1.6)

$$
\lim _{n \rightarrow \infty} \frac{n \sum_{j=1}^{n} r\left(\left|s_{n}-j\right|\right)}{\sum_{i=1}^{n} \sum_{j=1}^{n} r(|i-j|)} \geq \underline{\lim } \frac{n}{\left[\epsilon_{n} n\right]}\left(\sum_{k=1}^{n} \frac{1}{k}\right)^{-1}=\infty .
$$

Thus, we see that one can find no sequence of intervals $I_{n}$ satisfying (1.2) for which (1.3) holds. One also readily computes that

$$
r(0)=1 \text { and } r(n)=-\frac{1}{2} p_{n}=\frac{-1}{2 n(n+1)}, \quad n \geq 1,
$$

so that (1.1) holds.

Remark. Although we obtained our correlation function as a mixture of correlation functions making evident that the function defined in (1.4) is a correlation function, it is also easily checked directly that $r$ defined in (1.8) is nonnegative definite for any probability mass function $p_{n}$.

In section section2, we show the asymptotic independence of $\left(S_{n}, M_{n}\right)$ provided $M_{n}$ has a nondegenerate limit and $\frac{\log n}{n} \sum_{i=1}^{n}|r(i)|=o(1)$ as $n \rightarrow \infty$, a condition weaker than (1.1) so that, in particular, asymptotic independence holds in the case of a stationary Gaussian sequence with correlation function given in (1.8). Thus, the Ho and Hsing (1996) paper has a gap. A similar short coming exists in the Ho and McCormick (1999) paper.

As the example suggests the earlier work on this problem has difficulty with sequences for which $\operatorname{Var}\left(S_{n}\right)=\sum_{i=1}^{n} \sum_{j=1}^{n} r(|i-j|)$ grows too slowly or, more precisely, with variables 
$X_{s}$ such that the quantity, $E X_{s} \bar{X}_{n} / E\left(\bar{X}_{n}\right)^{2}$, is too large. We overcome this difficulty by the devise of deleting problematical variables in a precise way and establishing that the variables so culled form a negligible subset compared to the variables retained.

To conclude the introduction, we remark that while the present paper and Ho and McCormick (1999) address similar questions, the techniques used in the two papers are quite different. The latter paper's main focus is an analysis of the distribution of the maximum conditional on the value of the sum while in this paper an essential step is the construction of an intermediary sequence sufficiently close to the original sequence but constructed in such a way that the intermediary sequence belongs to the space of variables independent to the sum.

\section{Asymptotic Independence}

In this section we shall establish the asymptotic independence of the partial sums and partial maxima of Gaussian sequences. For future applications, it will be convenient to present results for triangular arrays of Gaussian sequences. Therefore, for $n \geq 1$, let $\left\{X_{n i}, 1 \leq\right.$ $i \leq n\}$ be a Gaussian sequence, i.e. all joint distributions are Gaussian. We assume the variables are centered, i.e.,

$$
E X_{n i}=0, \quad 1 \leq i \leq n, \quad n \geq 1
$$

Further, setting $\sigma_{n}(i, j)=E X_{n i} X_{n j}, 1 \leq i, j \leq n$, we shall assume that

$$
\max _{1 \leq i \leq n}\left|1-\sigma_{n}(i, i)\right|=0\left(\frac{1}{\log n}\right) \text { as } n \rightarrow \infty .
$$

The following mixing condition for the array will be suitable

$$
\lim _{n \rightarrow \infty} \frac{\log n}{n^{2}} \sum_{i=1}^{n} \sum_{j=1}^{n}\left|\sigma_{n}(i, j)\right|=0 .
$$

Furthermore, put

$$
a_{n}=\sqrt{2 \log n} \text { and } b_{n}=a_{n}-\left(2 a_{n}\right)^{-1} \log (4 \pi \log n),
$$

For $I \subset[1, n]$ set

$$
M_{n}(I)=\max _{i \in I} X_{n i} \text { and } S_{n}(I)=\sum_{i \in I} X_{n i}
$$

In the case $I=[1, n]$, we also put

$$
M_{n}=M([1, n])=\max _{1 \leq i \leq n} X_{n i} \text { and } S_{n}=S([1, n])=\sum_{i=1}^{n} X_{n i}
$$


Our first lemma establishes a suitable subset of the index set which asymptotically determines the distribution of the maximum. To that end, fix a sequence of integers $m(n), n \geq 1$ satisfying

$$
\lim _{n \rightarrow \infty} m(n)=\infty
$$

and

$$
\lim _{n \rightarrow \infty} \frac{m(n) \log n}{n^{2}} \sum_{i=1}^{n} \sum_{j=1}^{n}\left|\sigma_{n}(i, j)\right|=0 .
$$

Let

$$
\delta_{n}(i)=E X_{n i} S_{n}=\sum_{j=1}^{n} \sigma_{n}(i, j), \quad 1 \leq i \leq n,
$$

and define sets

$$
I_{n}^{+}=\left\{i: \delta_{n}(i) \geq 0, \quad 1 \leq i \leq n\right\}
$$

and

$$
I_{n}^{-}=\left\{i: \delta_{n}(i)<0, \quad 1 \leq i \leq n\right\} .
$$

Note that $I_{n}^{+} \neq \phi$. Let $s_{n}^{+}$and $s_{n}^{-}$denote the sums, $s_{n}^{+}=\sum_{j \in I_{n}^{+}} \delta_{n}(j)$ and $s_{n}^{-}=\sum_{j \in I_{n}^{-}} \delta_{n}(j)$ if $I_{n}^{-} \neq \phi$. Next define

$$
K_{n}^{+}=\left\{i \in I_{n}^{+}: \frac{\delta_{n}(i)}{s_{n}^{+}} \geq \frac{\log m(n)}{n}\right\}
$$

and if $I_{n}^{-} \neq \phi$,

$$
K_{n}^{-}=\left\{i \in I_{n}^{-}: \frac{\delta_{n}(i)}{s_{n}^{-}} \geq \frac{\log m(n)}{n}\right\}
$$

Finally, set

$$
K_{n}=K_{n}^{+} \cup K_{n}^{-} \text {and } J_{n}=[1, n] \backslash K_{n}
$$

Lemma 2.1. Let $\left\{X_{n i}, 1 \leq i \leq n\right\}, n \geq 1$ be an array of Gaussian sequences such that (2.1) and (2.2) hold. Then, for $J_{n}$ defined in (2.7), we have

$$
P\left\{M_{n}\left(J_{n}\right) \leq u_{n}(y)\right\}-P\left\{M_{n} \leq u_{n}(y)\right\} \leq P\left\{M_{n}\left(K_{n}\right)>u_{n}(y)\right\} \rightarrow 0, \quad \text { as } n \rightarrow \infty,
$$

where $u_{n}(y)=b_{n}+\frac{y}{a_{n}}$ with $a_{n}$ and $b_{n}$ given in (2.4).

Proof. Observe that

$$
\begin{aligned}
& P\left\{M_{n}\left(J_{n}\right) \leq u_{n}(y)\right\} \\
= & P\left\{M_{n} \leq u_{n}(y)\right\}+P\left\{M_{n}\left(J_{n}\right) \leq u_{n}(y), M_{n}\left(K_{n}\right)>u_{n}(y)\right\} .
\end{aligned}
$$


Furthermore,

$$
\begin{aligned}
& P\left\{M_{n}\left(K_{n}\right)>u_{n}(y)\right\} \\
\leq & \#\left(K_{n}\right) \max _{1 \leq i \leq n} P\left\{X_{n i}>u_{n}(y)\right\} \\
= & \#\left(K_{n}\right)\left(1-\Phi\left(u_{n}(y) / \sigma_{n}^{*}\right)\right)
\end{aligned}
$$

where $\left[\sigma_{n}^{*}\right]^{2}=\max _{1 \leq i \leq n} \sigma_{n}(i, i)$ and $\Phi$ denotes the standard normal distribution function. Now observe that

$$
\#\left(K_{n}^{+}\right) \frac{\log m}{n} \leq \sum_{i \in K_{n}^{+}} \frac{\delta_{n}(i)}{s_{n}^{+}} \leq 1
$$

and similarly note that

$$
\#\left(K_{n}^{-}\right) \frac{\log m}{n} \leq 1
$$

where \# denotes cardinality and we put $m=m(n)$. Therefore,

$$
\#\left(K_{n}\right) \leq \frac{2 n}{\log m} .
$$

Therefore by $(2.2),(2.4),(2.9)$, and (2.10), we have

$$
P\left\{M_{n}\left(K_{n}\right)>u_{n}(y)\right\}=o(1) \text { as } n \rightarrow \infty,
$$

which proves the lemma in view of (2.8).

Our next step is to produce an intermediary array sufficiently close to the $\left\{X_{n i}\right\}$ array but independent of $S_{n}$. For that purpose we define for a subset $I \subset[1, n]$,

$$
\sigma_{n}^{2}(I)=\operatorname{Var}\left(S_{n}(I)\right)
$$

and in the case $I=[1, n]$ we put

$$
\sigma_{n}^{2}=\sigma_{n}^{2}([1, n])
$$

Then observe that

$$
\begin{aligned}
\sigma_{n}^{2}\left(I_{n}^{+}\right) & =\sum_{i, j \in I_{n}^{+}} E X_{n i} X_{n j} \\
& =\sum_{i, j \in I_{n}^{+}} \sigma_{n}(i, j) \\
& \leq \sum_{i=1}^{n} \sum_{j=1}^{n}\left|\sigma_{n}(i, j)\right| .
\end{aligned}
$$

Similarly, if $I_{n}^{-} \neq \phi$, we have

$$
\begin{aligned}
\sigma_{n}^{2}\left(I_{n}^{-}\right) & =\sum_{i, j \in I_{n}^{-}} E X_{n i} X_{n j} \\
& \leq \sum_{i=1}^{n} \sum_{j=1}^{n}\left|\sigma_{n}(i, j)\right| .
\end{aligned}
$$


For $i \in J_{n}$, define constants $c_{n}(i)$ by

$$
c_{n}(i)=\frac{\delta_{n}(i)}{s_{n}^{+}}, \quad i \in I_{n}^{+} \cap J_{n}
$$

and if $I_{n}^{-} \neq \phi$,

$$
c_{n}(i)=\frac{\delta_{n}(i)}{s_{n}^{-}}, \quad i \in I_{n}^{-} \cap J_{n} .
$$

We define our intermediary array $\left\{Y_{n i}, i \in J_{n}\right\}$ by

$$
Y_{n i}=X_{n i}-c_{n}(i) S_{n}\left(I_{n}^{+}\right) \text {if } i \in I_{n}^{+} \cap J_{n}
$$

and

$$
Y_{n i}=X_{n i}-c_{n}(i) S_{n}\left(I_{n}^{-}\right) \text {if } i \in I_{n}^{-} \cap J_{n} .
$$

Lemma 2.2. For a Gaussian array $\left\{X_{n i}, 1 \leq i \leq n, n \geq 1\right\}$ such that (2.1), (2.2), and (2.3) hold,

$$
a_{n}\left[M_{n}\left(J_{n}\right)-\max _{i \in J_{n}} Y_{n i}\right] \stackrel{L^{1}}{\longrightarrow} 0,
$$

where $J_{n}$ is defined in (2.7) and $Y_{n i}$ in (2.13).

Proof. By virtue of the inequality

$$
\left|\max _{1 \leq j \leq n} x_{j}-\max _{1 \leq j \leq n} y_{j}\right| \leq \max _{1 \leq j \leq n}\left|x_{j}-y_{j}\right|
$$

one has that

$$
\begin{aligned}
\left|M_{n}\left(J_{n}\right)-\max _{i \in J_{n}} Y_{n i}\right| & \leq \max _{i \in J_{n}}\left|c_{n}(i)\right|\left[\left|S_{n}\left(I_{n}^{+}\right)\right|+\left|S_{n}\left(I_{n}^{-}\right)\right|\right] \\
& \leq \frac{\log m}{n}\left[\left|S_{n}\left(I_{n}^{+}\right)\right|+\left|S_{n}\left(I_{n}^{-}\right)\right|\right] .
\end{aligned}
$$

Therefore by (2.14) and Cauchy-Schwarz inequality

$$
\begin{aligned}
& E\left|M_{n}\left(J_{n}\right)-\max _{i \in J_{n}} Y_{n i}\right| \\
\leq & \frac{\log m}{n}\left[\sigma_{n}\left(I_{n}^{+}\right)+\sigma_{n}\left(I_{n}^{-}\right)\right] \\
\leq & \frac{2 \log m}{n}\left[\sum_{i=1}^{n} \sum_{j=1}^{n}\left|\sigma_{n}(i, j)\right|\right]^{1 / 2} \\
= & o\left(a_{n}^{-1}\right),
\end{aligned}
$$

where we used (2.11), (2.12), and properties (2.5) and (2.6) of the sequence $m$. 
Theorem 2.3. Under the hypothesis of Lemma 2.2 if for some distribution function $G$,

$$
a_{n}\left(M_{n}-b_{n}\right) \stackrel{d}{\longrightarrow} G
$$

then

$$
\lim _{n \rightarrow \infty} P\left\{\frac{S_{n}}{\sigma_{n}} \leq x, \quad a_{n}\left(M_{n}-b_{n}\right) \leq y\right\}=\Phi(x) G(y)
$$

for all $-\infty<x<\infty$ and continuity points $y$ of $G$.

Proof. We first observe that for $i \in J_{n}$

$$
E Y_{n i} S_{n}=0
$$

Therefore, since $\left(Y_{n i}, S_{n}\right)$ have a joint normal distribution, the variables are independent. Now observe that, for any real $x$ and continuity point $y$ of $G$,

$$
\begin{aligned}
& \left|P\left\{\frac{S_{n}}{\sigma_{n}} \leq x, a_{n}\left(M_{n}-b_{n}\right) \leq y\right\}-\Phi(x) G(y)\right| \\
\leq & \left|P\left\{\frac{S_{n}}{\sigma_{n}} \leq x, M_{n} \leq u_{n}(y)\right\}-P\left\{\frac{S_{n}}{\sigma_{n}} \leq x, M_{n}\left(J_{n}\right) \leq u_{n}(y)\right\}\right| \\
+ & \left|P\left\{\frac{S_{n}}{\sigma_{n}} \leq x, M_{n}\left(J_{n}\right) \leq u_{n}(y)\right\}-P\left\{\frac{S_{n}}{\sigma_{n}} \leq x, \max _{i \in J_{n}} Y_{n i} \leq u_{n}(u)\right\}\right| \\
+ & \left|P\left\{\frac{S_{n}}{\sigma_{n}} \leq x, \max _{i \in J_{n}} Y_{n i} \leq u_{n}(y)\right\}-\Phi(x) G(y)\right| \\
= & T_{n, 1}+T_{n, 2}+T_{n, 3} .
\end{aligned}
$$

Now observe that by Lemma 2.1

$$
\begin{aligned}
T_{n, 1} & \leq E\left|I\left\{M_{n} \leq u_{n}(y)\right\}-I\left\{M_{n}\left(J_{n}\right) \leq u_{n}(y)\right\}\right| \\
& \leq P\left\{M\left(K_{n}\right)>u_{n}(y)\right\}=o(1) \text { as } n \rightarrow \infty .
\end{aligned}
$$

Also,

$$
\begin{aligned}
& T_{n, 2} \\
\leq & P\left\{y-a_{n}\left|M_{n}\left(J_{n}\right)-\max _{i \in J_{n}} Y_{n i}\right| \leq a_{n}\left(M_{n}\left(J_{n}\right)-b_{n}\right) \leq y+a_{n}\left|M_{n}\left(J_{n}\right)-\max _{i \in J_{n}} Y_{n i}\right|\right\} \\
= & o(1) \text { as } n \rightarrow \infty
\end{aligned}
$$

where we used Lemma 2.2, (2.15) and (2.18).

Finally note that in view of (2.16) and the consequent independence

$$
T_{n, 3}=\Phi(x)\left|P\left\{\max _{i \in J_{n}} Y_{n i} \leq u_{n}(y)\right\}-G(y)\right|=o(1) \text { as } n \rightarrow \infty
$$

by Lemma 2.2 and (2.18). Thus the result follows from (2.17)-(2.20). 
Remark: Consider the case of a stationary Gaussian sequence, $\left\{X_{k}\right\}$. Putting $X_{n k}=X_{k}$ in the previous result, we see that condition (2.3) is equivalent to the condition

$$
\lim _{n \rightarrow \infty} \frac{\log n}{n} \sum_{i=1}^{n}|r(i)|=0,
$$

where $r(i)=E X_{1} X_{i+1}=\sigma_{n}(j, i+j)$. It is not known if the above condition implies (2.15). See Leadbetter et al. (1983) p 91. Thus, the condition (2.15) is not superfluous.

Corollary 2.4. Let $\left\{X_{n}, n \geq 1\right\}$ be a Gaussian sequence such that $E X_{n}=0, E X_{n}^{2}=1$, $n \geq 1$. Suppose there exists a sequence $\rho_{n}, n \geq 0$ with $\rho_{n}<1, n \geq 1$ and

$$
\left|E X_{i} X_{j}\right| \leq \rho_{|i-j|}
$$

such that $\rho_{n} \log n \rightarrow 0$ as $n \rightarrow \infty$. Then, for $-\infty<x, y<\infty$,

$$
\lim _{n \rightarrow \infty} P\left\{\frac{1}{\sigma_{n}} S_{n} \leq x, \quad a_{n}\left(M_{n}-b_{n}\right) \leq y\right\}=\Phi(x) \exp \left(-e^{-y}\right),
$$

where $S_{n}=\sum_{i=1}^{n} X_{i}$ and $M_{n}=\max _{1 \leq i \leq n} X_{i}$.

Proof. By Theorem 6.3.4 in Leadbetter et al. (1983)

$$
\lim _{n \rightarrow \infty} P\left\{a_{n}\left(M_{n}-b_{n}\right) \leq y\right\}=\exp \left(-e^{-y}\right)=: \Lambda(y) .
$$

Then as the hypothesis of Theorem 2.3 is readily verified for the array $X_{n i}=X_{i}, 1 \leq i \leq$ $n, n \geq 1$, the result follows from Theorem 2.3 and (2.21).

The following elementary result on series will prove useful.

Lemma 2.5. Let $\left\{c_{n k}\right\}$ be a bounded array of nonnegative reals. Then the following two statements are equivalent.

$$
\lim _{n \rightarrow \infty} \frac{\log n}{n} \sum_{k=1}^{n} c_{n k}=0
$$

and

$$
\lim _{n \rightarrow \infty} \frac{1}{n} \sum_{k=1}^{n} c_{n k} \log k=0 .
$$

Proof. Let $c$ be an upper bound for the array. Let $j_{n}=\left[n /(\log n)^{2}\right]$. Then note for all $n$ large

$$
\log n<2 \log k, \quad j_{n}<k \leq n
$$


Suppose (2.23) holds. Then

$$
\begin{aligned}
& \lim _{n \rightarrow \infty} \frac{\log n}{n} \sum_{k=1}^{n} c_{n k} \\
= & \lim _{n \rightarrow \infty}\left(\frac{\log n}{n} \sum_{k=1}^{j_{n}} c_{n k}+\frac{\log n}{n} \sum_{k=j_{n}+1}^{n} c_{n k}\right) \\
\leq & \lim _{n \rightarrow \infty} \frac{c j_{n} \log n}{n}+\lim _{n \rightarrow \infty} \frac{2}{n} \sum_{k=j_{n}+1}^{n} c_{n k} \log k=0 .
\end{aligned}
$$

Since (2.23) follows a fortiori from (2.22), the lemma follows from (2.24).

The following corollary relates to stationary sequences. The mixing condition imposed is a weak sufficient condition to imply extremal index 1 for Gaussian stationary sequences.

Corollary 2.6. Let $\left\{X_{n}, n \geq 1\right\}$ be a stationary Gaussian sequence with $E X_{n}=0$ and $E X_{n}^{2}=1$. Let $r_{n}=E X_{1} X_{n+1}$. Suppose that

$$
r_{n}=o(1) \text { as } n \rightarrow \infty
$$

and, for some $\gamma>2$,

$$
\frac{1}{n} \sum_{k=1}^{n}\left|r_{k}\right| \log k \exp \left(\gamma\left|r_{k}\right| \log k\right)=o(1) \quad \text { as } n \rightarrow \infty .
$$

Then, for $-\infty<x, y<\infty$

$$
\lim _{n \rightarrow \infty} P\left\{\frac{1}{\sigma_{n}} S_{n} \leq x, a_{n}\left(M_{n}-b_{n}\right) \leq y\right\}=\Phi(x) \Lambda(y) .
$$

Proof. From (2.25), it follows trivially that

$$
\frac{1}{n} \sum_{k=1}^{n}\left|r_{k}\right| \log k=o(1) \text { as } n \rightarrow \infty .
$$

Now with $c_{n k}=\left|r_{k}\right|$, it follows from (2.26) and Lemma 2.5 that

$$
\frac{\log n}{n} \sum_{k=1}^{n}\left|r_{k}\right|=o(1) \text { as } n \rightarrow \infty .
$$

Thus by (2.27), Theorem 2.3, and Theorem 4.5.2 in Leadbetter et al. (1983), the corollary follows.

The previous corollaries relate to Gaussian stationary sequence, which are weakly dependent in the sense that they possess an extremal index equal to 1 . The next result pertains to the case of strongly dependent stationary Gaussian sequences for which an extremal index does not exist. See Leadbetter et al. (1983) p 133 for a presentation on limit 
laws for extremes for strongly dependent stationary Gaussian sequences. By considering $M_{n}-\bar{X}_{n}$ where $\bar{X}_{n}=\frac{1}{n} S_{n}$, one can derive the asymptotic behavior of $\left(S_{n}, M_{n}\right)$ from that of $\left(S_{n}, M_{n}-\bar{X}_{n}\right)$. However, as will be shown, whereas $\left\{X_{k}, k \geq 1\right\}$ may be strongly dependent, the array $\left\{X_{k}-\bar{X}_{n}, 1 \leq k \leq n, n \geq 1\right\}$ will fall within the scope of our Theorem 2.3. The following result generalizes Theorem 2.1 in Ho and McCormick (1999).

Theorem 2.7. Let $\left\{X_{k}, k \geq 1\right\}$ be a stationary Gaussian sequence with $E X_{k}=0, E X_{k}^{2}=$ 1 , and $r(k)=E X_{1} X_{k+1}$ satisfying

$$
r(k)<1 \text { for some } k \geq 1
$$

and

$$
\lim _{n \rightarrow \infty} \frac{\log n}{n} \sum_{k=1}^{n-1}|r(k)-r(n)|=0 .
$$

Then, for $-\infty<x, y<\infty$,

$$
\lim _{n \rightarrow \infty} P\left\{\frac{S_{n}}{\sigma_{n}} \leq x, a_{n}\left(\frac{M_{n}-\bar{X}_{n}}{\sqrt{1-r(n)}}-b_{n}\right) \leq y\right\}=\Phi(x) \Lambda(y) .
$$

Proof. We first remark that as shown in (2.7) of McCormick (1980), (2.28) and (2.29) imply

$$
\sup _{n \geq 1}|r(n)|<1 .
$$

Now let $\sigma_{n}^{2}(k)=E\left(X_{k}-\bar{X}_{n}\right)^{2}$ and set

$$
Y_{n k}=\frac{1}{\sigma_{n}(k)}\left(X_{k}-\bar{X}_{n}\right) .
$$

Then by (2.8) and (2.9) in McCormick (1980)

$$
\max _{1 \leq k \leq n}\left|\sigma_{n}^{2}(k)-\left(1-r_{n}\right)\right|=o\left(\frac{1}{\log n}\right)
$$

and

$$
\max _{1 \leq i<j \leq n}\left|E Y_{n i} Y_{n j}-\frac{r(i-j)-r(n)}{1-r(n)}\right|=o\left(\frac{1}{\log n}\right) .
$$

Now define

$$
X_{n i}=\frac{X_{i}-\left(\frac{n-1}{n}\right) \bar{X}_{n}}{\sqrt{1-r(n)}}
$$

Observe that by $(2.31)$

$$
\begin{aligned}
E X_{n i}^{2} & =\frac{\sigma_{n}^{2}(i)}{1-r(n)}\left(1+\frac{1}{2 n \sigma_{n}(i)} E\left(X_{i}-\bar{X}\right) \bar{X}+\frac{1}{n^{2}} E \bar{X}^{2}\right) \\
& =1+o\left(\frac{1}{\log n}\right)
\end{aligned}
$$


where the $o\left(\frac{1}{\log n}\right)$ term does not depend on $i$.

Moreover, by (2.31) and (2.32),

$$
\begin{aligned}
E X_{n i} X_{n j} & =\frac{\sigma_{n}(i) \sigma_{n}(j)}{1-r(n)} E Y_{n i} Y_{n j}+\frac{1}{(1-r(n)) n} E \bar{X}_{n}\left[X_{i}+X_{j}-2 \bar{X}_{n}\right] \\
& +\frac{1}{(1-r(n)) n^{2}} E \bar{X}_{n}^{2} \\
& =\frac{r(i-j)-r(n)}{1-r(n)}+o\left(\frac{1}{\log n}\right)
\end{aligned}
$$

where the $o\left(\frac{1}{\log n}\right)$ term does not depend on $i, j$.

Then setting

$$
\sigma_{n}(i, j)=E X_{n i} X_{n j}
$$

one readily checks by virtue of (2.33) that (2.2) holds. Of course, (2.1) holds. By (2.29), (2.30) and (2.34) one obtains

$$
\begin{aligned}
& \sum_{i=1}^{n} \sum_{j=1}^{n}\left|\sigma_{n}(i, j)\right| \\
= & \sum_{i=1}^{n} \sum_{j=1}^{n}\left|\frac{r(i-j)-r(n)}{1-r(n)}\right|+o\left(\frac{n^{2}}{\log n}\right) \\
\leq & \frac{n}{1-r(n)} \sum_{k=1}^{n}|r(k)-r(n)|+o\left(\frac{n^{2}}{\log n}\right) \\
= & o\left(\frac{n^{2}}{\log n}\right) .
\end{aligned}
$$

Therefore condition (2.3) holds.

Next observe that

$$
\begin{aligned}
& a_{n} E\left|\frac{M_{n}-\bar{X}_{n}}{\sqrt{1-r(n)}}-\max _{1 \leq i \leq n} X_{n i}\right| \\
\leq & \frac{a_{n}}{n(1-r(n))} E\left|\bar{X}_{n}\right| \\
\leq & \frac{a_{n}}{n(1-r(n))}=o(1) \text { as } n \rightarrow \infty .
\end{aligned}
$$

Therefore, since by Theorem 2.1 in McCormick (1980)

$$
a_{n}\left(\frac{M_{n}-\bar{X}_{n}}{\sqrt{1-r(n)}}-b_{n}\right) \stackrel{d}{\longrightarrow} \Lambda,
$$

we have by (2.35) and (2.36) that

$$
a_{n}\left(\max _{1 \leq i \leq n} X_{n i}-b_{n}\right) \stackrel{d}{\longrightarrow} \Lambda .
$$


Now observe that

$$
\sum_{i=1}^{n} X_{n i} / \sqrt{\operatorname{Var}\left(\sum_{i=1}^{n} X_{n i}\right)}=\frac{(1 / n) \bar{X}_{n}}{\sqrt{\operatorname{Var}\left(1 / n \bar{X}_{n}\right)}}=\frac{S_{n}}{\sigma_{n}} .
$$

Thus by (2.37) and (2.38), the result follows from Theorem 2.3.

The following corollary generalizes Corollary 2.2 in Ho and McCormick (1999). Since the method of proof is the same, we present just the result.

Corollary 2.8. Suppose $\left\{X_{k}, k \geq 1\right\}$ is a stationary Gaussian sequence with $E X_{k}=0$, $E X_{k}^{2}=1$ and $r(k)=E X_{1} X_{k+1}$ satisfying

$$
r(k) \log k \rightarrow \gamma \in[0, \infty), \quad \text { as } k \rightarrow \infty
$$

Then, for $-\infty<x, y<\infty$,

$$
\lim _{n \rightarrow \infty} P\left\{\frac{S_{n}}{\sigma_{n}} \leq x, a_{n}\left(M_{n}-b_{n}\right) \leq y\right\}=\int_{-\infty}^{x} \exp \left\{-e^{-y+\gamma-\sqrt{2 \gamma} z)}\right\} \phi(z) d z
$$

where $\phi(z)=\frac{1}{\sqrt{2 \pi}} e^{-1 / 2 z^{2}}$.

In the case of divergence in (2.39), convergence of the joint distribution of $\left(S_{n}, M_{n}\right)$ occurs but to a singular limit distribution.

Corollary 2.9. Let $\left\{X_{k}, k \geq 1\right\}$ be a stationary Gaussian sequence with $E X_{k}=0, E X_{k}^{2}=$ 1 and $r(k)=E X_{1} X_{k+1}$ satisfying

$$
r(k) \log k \rightarrow \infty, \quad \text { as } k \rightarrow \infty,
$$

and either

(a) conditions (2.28) and (2.29) hold

or

(b) (i) $r(n) \rightarrow 0$ and is monotonically nonincreasing for $n \geq n_{0}$ for some positive integer $n_{0}$ and (ii) $r(n) \log n$ is monotonically nondecreasing for $n \geq n_{0}$.

Then

$$
\lim _{n \rightarrow \infty} P\left\{\frac{S_{n}}{\sigma_{n}} \leq x, r(n)^{-1 / 2}\left(\frac{M_{n}}{\sqrt{1-r(n)}}-b_{n}\right) \leq y\right\}=\Phi(x \wedge(y \sqrt{1-\delta}))
$$

where $\delta=\lim _{n \rightarrow \infty} r(n)$. 
Proof. Case (a) is done in Ho and McCormick (1999). For case (b), observe that

$$
\begin{aligned}
& \frac{1}{n} \sum_{k=1}^{n}|r(k)-r(n)| \log k \\
= & \frac{1}{n} \sum_{k=1}^{n_{0}}|r(k)-r(n)| \log k+\frac{1}{n} \sum_{k=n_{0}+1}^{n}|r(k)-r(n)| \log k \\
= & o(1)+\frac{1}{n} \sum_{k=n_{0}+1}^{n}(r(k)-r(n)) \log k \\
\leq & o(1)+\frac{1}{n} \sum_{k=n_{0}+1}^{n}(r(n) \log n-r(n) \log k) \\
\leq & o(1)+r(n) \int_{0}^{1}(-\log x) d x \\
= & o(1)+r(n) .
\end{aligned}
$$

Thus by (2.40) and Lemma 2.5, we see that (2.29) holds.

Now under the hypotheses in case (b), we have by McCormick and Mittal (1976) that

$$
r(n)^{-1 / 2}\left(M_{n}-\sqrt{1-r(n)} b_{n}\right) \stackrel{d}{\longrightarrow} \Phi .
$$

Then the proof finishes as in Ho and McCormick (1999).

Remark: As observed in Corollories 2.8 and 2.9, in the strongly dependent case the limit law or the normalization or both depend on the correlation sequence. For that reason, it may be advantageous to model using the studentized maxima. In addition to the quantity $\frac{M_{n}-\bar{X}_{n}}{s_{n}}$, where $s_{n}^{2}=\frac{1}{n} \sum_{1}^{n}\left(X_{k}-\bar{X}_{n}\right)^{2}$, being invariant under a scale and location transformation of the underlying sequence, its asymptotic behavior remains the same whether or not the dependence is strong or weak. More precisely, in view of Lemma 2.2 in McCormick (1980),

$$
\log n\left[s_{n}^{2}-(1-r(n))\right] \stackrel{L^{2}}{\longrightarrow} 0
$$

under the hypothesis of Theorem 2.7 , so that $\sqrt{1-r(n)}$ may be replaced by $s_{n}$ in the conclusion of that result.

\section{Continuous Time Processes}

In this section we present a sketch of the proof of Theorem 2.7 in the continuous time setting. Let $\left\{X_{t}, t \geq 0\right\}$ be a continuous time stationary Gaussian process with $E X_{t}=0$, $E X_{t}^{2}=1$, and $r(t)=E X_{0} X_{t}$. Suppose for some $c>0$ and $0<\alpha \leq 2$,

$$
r(t)=1-c|t|^{\alpha}+o\left(|t|^{\alpha}\right) \text { as } t \rightarrow 0 .
$$


Then $\left\{X_{t}, t \geq 0\right\}$ has a version with continuous sample paths. Define

$$
M_{T}=\max _{0 \leq t \leq T} X_{t}, S_{T}=\int_{0}^{T} X_{t} d t \text { and } \bar{X}_{T}=\frac{1}{T} S_{T}
$$

Let $\{Y(t)\}$ denote a nonstationary Gaussian process with

$$
E Y(t)=-|t|^{\alpha} \text { and } \operatorname{Cov}(Y(s), Y(t))=|s|^{\alpha}+|t|^{\alpha}-|s-t|^{\alpha} .
$$

Define a positive finite constant (See Leadbetter et al. (1983) p 232) by

$$
H_{\alpha}=\lim _{T \rightarrow \infty} T^{-1} \int_{-\infty}^{0} e^{-x} P\left\{\max _{0 \leq t \leq T} Y(t)>-x\right\} d x .
$$

Let

$$
a_{T}=(2 \log T)^{1 / 2}
$$

and

$$
b_{T}=a_{T}+\frac{1}{a_{T}}\left\{\left(\frac{1}{\alpha}-\frac{1}{2}\right) \log \log T+\log \left([2 \pi]^{-1 / 2}\left(\frac{c}{1-\gamma}\right)^{1 / \alpha} H_{\alpha} 2^{\left(\frac{2-\alpha}{2 \alpha}\right)}\right)\right\}
$$

where $\gamma=$ atom at zero of the spectral distribution associated with $r$ in (3.1). Note under (3.2) below, it can be shown that $\gamma=\lim _{T \rightarrow \infty} r(T)$. The following result generalizes Theorem 3.1 in Ho and McCormick (1999).

Theorem 3.1. Let $\{X(t)\}$ be a stationary Gaussian process such that $E X(t)=0$, $E X^{2}(t)=1$ and $r(t)=E X_{0} X_{t}$ satisfies (3.1). Further assume

$$
\lim _{T \rightarrow \infty} \frac{\log T}{T} \int_{0}^{T}|r(t)-r(T)| d t=0 .
$$

Then

$$
\lim _{T \rightarrow \infty} P\left\{\frac{S_{T}}{\sigma_{T}} \leq x, a_{T}\left(\frac{M_{T}-\bar{X}_{T}}{\sqrt{1-r(T)}}-b_{T}\right) \leq y\right\}=\Phi(x) \Lambda(y),
$$

$-\infty<x, y<\infty$ where $\sigma_{T}^{2}=\operatorname{Var}\left(S_{T}\right)$.

Proof. We obtain a discretization set as follows. For $\epsilon>0$ there exists a $\tau>0$ such that for all large $T$

$$
\frac{1-\epsilon}{1-\gamma} c|s-t|^{\alpha}<1-\rho_{T}(s, t)<\frac{1+\epsilon}{1-\gamma} c|s-t|^{\alpha} \text { for }|s-t| \leq \tau
$$

where $\rho_{T}(s, t)=E Y_{T}(s) Y_{T}(t)$ with $Y_{T}(t)=\frac{1}{\sigma_{T}(t)}\left(X_{t}-\bar{X}_{T}\right)$ and $\sigma_{T}^{2}(t)=E\left(X_{t}-\bar{X}_{T}\right)^{2}$. For a $\tau$ for which (3.4) is valid and $0<a<\tau$, let $I_{k}=[k \tau+a,(k+1) \tau]$ and $I(T)=\bigcup_{k} I_{k} \cap[0, T]$. Set for fixed $y$

$$
u_{T}(y)=b_{T}+y / a_{T}
$$


and define a discretization set by

$$
G(T, a)=\left\{s \in I(T): s=a k u_{T}^{-2 / \alpha}, k=0,1, \ldots\right\}
$$

By the proof of Theorem 3.1 in McCormick (1980), one can check that to establish the result it suffices to show

$$
\lim _{a \downarrow 0} \lim _{T \rightarrow \infty} P\left\{\frac{S_{T}}{\sigma_{T}} \leq x, \max _{s \in G(T, a)} Y_{T}(s) \leq u_{T}(y)\right\}=\Phi(x) \Lambda(y) .
$$

Finally, (3.5) is established by following the method of proof of Theorem 2.7 and Theorem 3.1 in McCormick (1980).

\section{References}

Chow, T. L. and Teugels, J. L. (1978). The sum and the maximum of i.i.d. random variables. In Proc. Second Prague Symp. Asymptotic Statistics, North Holland, N.Y.

Ho, H. C. and Hsing, T. (1996). On the asymptotic joint distribution of the sum and maximum of stationary normal random variables. J. Appl. Probab. 33, 138-145.

Ho, H. C. and McCormick, W. P. (1999). Asymptotic distribution of sum and maximum for Gaussian processes. J. Appl. Probab. 36, 1031-1044.

Hsing, T. (1995). A note on the asymptotic independence of the sum and maximum of strongly mixing stationary random variables. Ann. Probab. 23, 938-947.

Leadbetter, M. R., Lindgren, G. and Rootzen, H. (1983). Extremes and Related Properties of Stationary Sequences and Processes. Springer-Verlay, N. Y.

McCormick, W. P. (1980). Weak convergence for the maxima of stationary Gaussian processes using random normalization. Ann. Probab. 8, 483-497.

McCormick, W. P. and Mittal, Y. (1976). On weak convergence of the maximum. Tech Report No 81, Dept. of Statist., Stanford Univ. 